\title{
Odontoid screw placement for Anderson type II odontoid fractures: how do duration from injury to surgery and clinical and radiological factors influence the union rate? A multicenter retrospective study
}

\author{
Luca Ricciardi, MD, ${ }^{1,2}$ Sokol Trungu, MD, ${ }^{1,2}$ Alba Scerrati, MD, PhD, ${ }^{3}$ Pasquale De Bonis, MD, PhD, ${ }^{3}$ \\ Oriela Rustemi, MD, ${ }^{4}$ Mauro Mazzetto, MD, ${ }^{4}$ Giorgio Lofrese, MD, ${ }^{5}$ Francesco Cultrera, MD, ${ }^{5}$ \\ Cédric Y. Barrey, MD, PhD, ${ }^{6}$ Alessandro Di Bartolomeo, MD, ${ }^{2,6}$ Amedeo Piazza, MD, ${ }^{6}$ \\ Massimo Miscusi, MD, $\mathrm{PhD},{ }^{6}$ and Antonino Raco, MD, $\mathrm{PhD}^{6}$ \\ 1 UO di Neurochirurgia, Ospedale Cardinal G. Panico, Tricase; '2UOC di Neurochirurgia, Azienda Ospedaliera Sant'Andrea, \\ Sapienza, Rome; ${ }^{3}$ Dipartimento di Neurochirurgia, Azienda Ospedaliero, Universitaria S. Anna, Ferrara; ${ }^{4}$ UOC di Neurochirurgia,

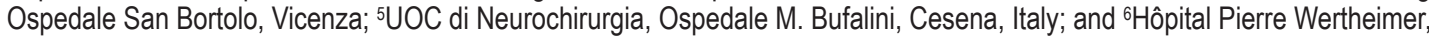 \\ Lyon, France
}

OBJECTIVE Anderson type II odontoid fractures are severe conditions, mostly affecting elderly people ( $\geq 70$ years old). Surgery can be performed as a primary treatment or in cases of failed conservative management. This study aimed to investigate how duration from injury to surgery, as well as clinical, radiological, and surgical risk factors, may influence the union rate after anterior odontoid screw placement for Anderson type II odontoid fractures.

METHODS The authors conducted a retrospective multicenter study. Demographic, clinical, surgical, and radiological data of patients who underwent anterior odontoid screw placement for Anderson type II fractures were retrieved from institutional databases. Study exclusion criteria were prolonged corticosteroid drug therapy (> 4 weeks), polytraumatic injuries, oncological diagnosis, and prior cervical spine trauma.

RESULTS Eighty-five patients were included in the present investigation. The union rate was $76.5 \%$, and 73 patients $(85.9 \%)$ did not report residual instability. Age $\geq 70$ years $(p<0.001$, OR 6$)$, female gender $(p=0.016$, OR 3.61), osteoporosis $(p=0.009$, OR 4.02), diabetes $(p=0.056$, OR 3.35), fracture diastasis $>1 \mathrm{~mm}(p<0.001$, OR 8.5$)$, and duration from injury to surgery $>7$ days $(p=0.002$, OR 48) independently influenced union rate, whereas smoking status $(p=$ $0.677, \mathrm{OR} 1.24)$ and odontoid process angulation $>10^{\circ}(p=0.885$, OR 0.92) did not.

CONCLUSIONS Although many factors have been reported as influencing the union rate after anterior odontoid screw placement for Anderson type II fractures, duration from injury to surgery $>7$ days appears to be the most relevant, resulting in a 48 times higher risk for nonunion. Early surgery appears to be associated with better radiological outcomes, as reported by orthopedic surgeons in other districts. Prospective comparative clinical trials are needed to confirm these results.

https://thejns.org/doi/abs/10.3171/2020.6.SPINE20318

KEYWORDS odontoid; fracture; cervical spine; injury; trauma; early surgery; fusion

$\mathrm{T}$ He craniovertebral junction (CVJ) is involved in as many as $20 \%$ of cervical spine traumatic injuries. ${ }^{1-3}$ Odontoid fractures are mostly reported in the elderly, and they were classified by Anderson and D'Alonzo into 3 types. Odontoid type II fractures are those involving the base of the odontoid process, which may be displaced and/or angulated backward or forward. ${ }^{4}$ These fractures result in C1-2 segmental instability, which may provide bulbar-medullary compression and segmental deformity.,5
Both conservative and surgical management have been proposed for odontoid type II fractures. ${ }^{6-8}$ The anterior cervical approach for dens screw placement was first reported by Böhler in 1982; it has been increasingly preferred to posterior and anterior $\mathrm{C} 1-2$ fusion techniques due to its surgical advantages and to the possibility of preserving segmental range of motion. ${ }^{9-11}$ Achieving no residual instability has been proposed as the treatment goal after Anderson type II fractures.,12 Although often

ABBREVIATIONS CVJ = craniovertebral junction.

SUBMITTED March 7, 2020. ACCEPTED June 1, 2020.

INCLUDE WHEN CITING Published online October 2, 2020; DOI: 10.3171/2020.6.SPINE20318. 
no visible signs of fusion are recognizable on postoperative imaging, patients do not report segmental instability on dynamic studies. ${ }^{12}$ While surgery provides primary stability that should theoretically lead to secondary fusion, conservative treatments promote fracture healing using external orthoses for immobilization. ${ }^{13}$ In cases of failure of conservative management, delayed surgery may be needed.$^{14-16}$ However, comparative data on the relevance of timing for surgery are still missing.

This study aimed to investigate how age, gender, smoking status, diabetes, osteoporosis, duration from injury to surgery, fracture diastasis, and odontoid angulation may influence the union rate.

\section{Methods}

\section{Study Design and Participating Centers}

This is a retrospective study retrieving data prospectively collected from 5 Italian and French institutional databases. The IRB of Azienda Ospedaliera Sant'Andrea approved the retrieval of anonymized data from institutional databases for the present investigation.

\section{Inclusion and Exclusion Criteria}

The databases of the centers involved were screened from January 2008 to December 2017. Inclusion criteria were posttraumatic Anderson type II odontoid fracture, ${ }^{4}$ odontoid anterior screw placement as surgical treatment, complete clinical-radiological documentation, and minimum follow-up of 1 year. The exclusion criteria were prolonged corticosteroid drug therapy ( $>4$ weeks), polytraumatic injuries, oncological diagnosis, prior traumatic injuries, or cervical spine surgery.

\section{Data Collection and Outcome Measurement}

For every patient, the following data were prospectively collected: age, gender, comorbidities, smoking status, diabetes diagnosis, T-score for bone densitometry (dual-energy $\mathrm{x}$-ray absorptiometry scan; $<2.5$ was considered the cutoff for osteoporosis; examinations from the year prior to or performed in the 12 months following the injury were considered), treatment timing after injury, fracture diastasis (distance between fracture rims) and odontoid process angulation ${ }^{17}$ (odontoid deviation from the ideal alignment was considered as favorable or unfavorable according to its severity, $>10^{\circ}$ or $<10^{\circ}$ ) on the pre- and postoperative CT scans, follow-up length, union or nonunion status on $\mathrm{CT}$, and segmental stability or residual instability on dynamic radiographs at last follow-up evaluation. The radiological evaluation was performed by one senior radiologist from each contributing institution.

\section{Statistical Analysis}

Values were reported as mean \pm standard deviation. The Student t-test was used to compare the quantitative continuous variables. Fisher's exact test (2-sided) was used to compare categorical variables. Statistical significance was predetermined at an alpha level of 0.05. Univariate and multivariate multiple regression analyses were performed. StatPlus (AnalystSoft Inc.) was used for data analysis.

\section{Results}

\section{Patients Characteristics}

Five centers contributed to the present study. Two hundred twenty-seven cases were retrieved from institutional databases and 85 patients were ultimately included in the present investigation, according to the inclusion criteria. Posterior approaches $(\mathrm{n}=86,37.9 \%)$, multiple cervical fractures $(\mathrm{n}=21,9.3 \%)$, anterior $\mathrm{C} 1-2$ transarticular screw placement $(\mathrm{n}=16,7 \%)$, incomplete documentation $(\mathrm{n}=10,4.4 \%)$, and previous cervical trauma $(\mathrm{n}=9,4 \%)$ were the reasons for excluding the remaining 142 patients (62.6\%). The mean age of the included patients was 69.9 \pm 13.4 years (range 19-91 years), the male to female ratio was 3.05 (64 men to 21 women), 40 patients (47.1\%) were smokers, $18(21.2 \%)$ suffered from diabetes, and 20 (8 men and 12 women, 23.5\%) had osteoporosis. In terms of comorbidities, 18 (21.2\%) had arterial hypertension, 9 (10.6\%) had hypothyroidism, 4 (4.7\%) were cardiopathic, and $3(3.5 \%)$ suffered from rheumatoid arthritis. Patient characteristics and demographic data are summarized in Table 1.

\section{Surgical and Clinical Outcomes}

The mean duration from injury to surgery was $7.02 \pm$ 17.80 days, the mean fracture diastasis after surgery was $1.66 \pm 0.92 \mathrm{~mm}$, and odontoid process misalignment was $>10^{\circ}$ in 18 patients $(21.2 \%)$.

The 18 patients $(21.2 \%)$ who had a preoperative unfavorable fracture angulation also showed a postoperative angulation $>10^{\circ}$. Every patient wore a nonrigid cervical collar for 2-4 weeks after surgery. The mean follow-up duration was $16.45 \pm 5.69$ months. Twenty patients (23.5\%) did not achieve fracture healing; thus, the union rate was $76.5 \%$. In 73 patients (85.9\%), there was no residual instability. At the last follow-up visit, all patients with residual instability did not achieve bone union, whereas $12(60 \%)$ of the 20 patients who did not achieve segmental union also presented with residual instability.

In terms of complications, 5 patients (5.9\%) reported transitory dysphagia after surgery and completely recovered within 1 month. Peri-implant radiolucency was found on CT in 3 patients (3.5\%) as a sign of screw mobility and nonunion. One patient (1.2\%) died 14 months after surgery due to a heart attack.

\section{Subgroup Analysis}

A subgroup analysis was conducted to investigate how individual factors could influence the union rate. In the univariate analysis (Table 2 ), age $\geq 70$ years $(p<0.001$, OR 6), female gender ( $p=0.016$, OR 3.61), osteoporosis ( $p$ $=0.009$, OR 4.02), diabetes $(\mathrm{p}=0.056$, OR 3.35), fracture diastasis $>1 \mathrm{~mm}(\mathrm{p}<0.001$, OR 8.5), and duration from injury to surgery $>7$ days $(\mathrm{p}=0.002$, OR 48$)$ negatively influenced the union rate. Conversely, smoking status $(\mathrm{p}$ $=0.677, \mathrm{OR} 1.24)$ and odontoid process angulation $>10^{\circ}$ $(\mathrm{p}=0.885$, OR 0.92) did not appear to influence the union rate.

The multivariate analysis showed that age $\geq 70$ years ( $p$ $=0.0156)$, residual fracture diastasis $>1 \mathrm{~mm}(\mathrm{p}=0.0009)$, and duration from injury to surgery $>7$ days $(p=0.0235)$ 
TABLE 1. Patient characteristics and demographics

\begin{tabular}{lc}
\hline \multicolumn{1}{c}{ Variable } & Value \\
\hline Mean age \pm SD (range), yrs & $69.9 \pm 13.4(19-91)$ \\
\hline M/F ratio (no. of men/women) & $3.05(64 / 21)$ \\
\hline Smoking status, $\mathrm{n}(\%)$ & $40(47.1)$ \\
\hline Diabetes, $\mathrm{n}(\%)$ & $18(21.2)$ \\
\hline Osteoporosis, $\mathrm{n}(\mathrm{M} / \mathrm{F}, \%)$ & $20(8 / 12,23.5)$ \\
\hline Mean postop fracture diastasis $\pm \mathrm{SD}, \mathrm{mm}$ & $1.66 \pm 0.92$ \\
\hline Angulation $>10^{\circ}, \mathrm{n}(\%)$ & $18(21.2)$ \\
\hline Mean duration $\pm \mathrm{SD}$ from injury to surgery, days & $7.02 \pm 17.80$ \\
\hline Union, $\mathrm{n}(\%)$ & $65(76.5)$ \\
\hline Residual instability, $\mathrm{n}(\%)$ & $12(14.1)$ \\
\hline Mean follow-up duration $\pm \mathrm{SD}$, mos & $16.45 \pm 5.69$ \\
\hline
\end{tabular}

independently influenced the union rate, resulting in lower chances for union after Anderson type II fractures of the odontoid. Data from the univariate and multivariate analyses are summarized in Table 2.

\section{Discussion}

A consensus on the preferred treatment for Anderson type II odontoid fractures remains missing. $3,6-8,11,13,14,16,18,19$ An external orthosis is usually prescribed for conservative management; however, patient discomfort, reduced quality of life, risk for sarcopenia, residual neck pain, and restriction of the cervical range of motion should be carefully considered. ${ }^{20-22}$ Surgery provides primary stability, which increases the chances for secondary bone fusion, although this treatment is associated with specific risks. ${ }^{6-8,11}$ When compared with posterior approaches, anterior odontoid screw placement for Anderson type II fractures reported better surgical outcomes in terms of intraoperative blood loss and surgical duration, as well as lower risks for posterior approach-related complications such as CSF leak, vertebral artery injuries, and prone position-related risks, especially in elderly patients..$^{3,10,11,23}$ This represents a minimally invasive approach through the cervical fascial planes, which requires a mild soft-tissue retraction and preserves the $\mathrm{C} 1-2$ range of motion. ${ }^{9-11,24}$ However, it re- mains an ultra-specialist procedure that requires specific training and is associated with nonnegligible risks for vascular, nervous system, and dural injuries.

Anterior odontoid screw placement is supposed to result in bone union rather than fusion. Because aging unfavorably affects the union rate, and because this type of fracture is common in elderly people, surgeons have been progressively reconsidering posterior approaches for Anderson type II odontoid fractures. As previously proposed, achieving no residual instability might represent the treatment goal, ${ }^{1,12}$ although fusion provides stronger secondary stability. Accordingly, fusion should be always pursued, whereas no residual instability may represent a satisfactory suboptimal result. ${ }^{1,10,12,13}$ In contrast, anterior odontoid screw placement allows one to preserve the segmental mobility and obtain nonresidual instability, which could represent the best clinical-radiological outcome.

Over time, preserving the segmental mobility as close as possible to the native range of motion has been increasingly accepted as a pursuable outcome. Accordingly, because of previous investigations in which the superiority of fusion was not demonstrated in terms of clinical-radiological outcomes, preserving function has to be carefully evaluated when planning surgery.

This study, in a multicenter cohort of patients, aimed to investigate every factor influencing the union rate after odontoid screw placement for Anderson type II fractures. Our results showed that septuagenarian and octogenarian patients have an increased risk for nonunion, which was 6 times higher than in younger patients, thus confirming that age is a specific risk factor. ${ }^{17}$ Female gender and osteoporosis both appear to be risk factors for nonunion, associated with 3.61 and 4.02 times higher risks, respectively. Diabetes represented a specific risk factor, reporting an OR of 3.35. Interestingly, residual fracture diastasis $>1 \mathrm{~mm}$ after surgery was associated with an 8.5 times increased risk for nonunion, suggesting that noncomplete fracture reduction represents a specific risk factor, higher than preoperative diastasis $>5 \mathrm{~mm}$, as previously reported..$^{25}$ Moreover, the most influential factor was duration from injury to surgery $>7$ days, associated with a 48 times higher risk for nonunion. This appears to suggest that early surgery should always be preferred. Delayed surgery, due to either medical decision or failure of conservative management

TABLE 2. Univariate and multivariate analyses

\begin{tabular}{|c|c|c|c|c|c|}
\hline \multirow[b]{2}{*}{ Factors } & \multicolumn{3}{|c|}{ Univariate Analysis } & \multicolumn{2}{|c|}{ Multivariate Analysis } \\
\hline & OR & $95 \% \mathrm{Cl}$ & Significance & $95 \% \mathrm{Cl}$ & Significance \\
\hline Age $\geq 70$ yrs & 6 & -0.019 to -0.006 & $1.70233 \mathrm{E}^{-10 *}$ & -0.014 to -0.002 & $0.015582817^{*}$ \\
\hline Female & 3.61 & 0.464 to 0.049 & $0.015835208^{*}$ & -0.351 to 0.057 & 0.156609948 \\
\hline Smoking status & 1.24 & -0.225 to 0.147 & 0.677002316 & -0.192 to 0.117 & 0.631725618 \\
\hline Diabetes & 3.35 & -0.047 to -0.006 & 0.056089681 & -0.228 to 0.180 & 0.816284768 \\
\hline Osteoporosis & 4.02 & -0.490 to -0.071 & $0.00924612^{*}$ & -0.235 to 0.196 & 0.856776426 \\
\hline Diastasis >1 mm & 8.5 & -0.311 to -0.134 & $3.37373 \mathrm{E}^{-06 *}$ & -0.254 to -0.068 & $0.000942075^{*}$ \\
\hline Angulation $>10^{\circ}$ & 0.92 & -0.210 to 0.243 & 0.884638263 & -0.056 to 0.325 & 0.174493958 \\
\hline Timing $>7$ days & 48 & -0.013 to 0.003 & $0.001999444^{*}$ & -0.010 to -0.001 & $0.023525699^{*}$ \\
\hline
\end{tabular}


with external orthosis, may lead to higher chances for nonunion, and thus to a potential need for subsequent more invasive surgeries, such as posterior $\mathrm{Cl}-2$ arthrodesis. The multivariate analysis showed that age $\geq 70$ years, residual fracture diastasis $>1 \mathrm{~mm}$, and duration from injury to surgery $>7$ days represented independent risk factors for nonunion in this case series.

Early surgery has been already accepted as the standard of care in different traumatic injuries. ${ }^{26-29}$ Furthermore, a lower union rate has been reported in patients who underwent anterior odontoid screw placement for Anderson type II fractures $>18$ months after injury, when compared with those treated within 6 months. ${ }^{19,30}$ Factors such as blood supply of the fragment, prevention of callus formation, and local inflammation-promoting fracture healing ${ }^{28,29}$ could have been underestimated, or even not considered at all in previous investigations. Because the union rate in conservative management has been proved to be lower than the rate after surgery, ${ }^{19}$ and delayed surgeries appear to be associated with lower chances for union, early surgery should be always preferred. Conversely, conservative management should be reserved for carefully selected patients reporting higher risks for surgery.

Smoking status and residual angulation $>10^{\circ}$ after surgery did not influence the union rate in our case series. Fragment angulation and oblique-posterior displacement have been previously reported as specific risk factors: ${ }^{17}$ however, our results suggest that fracture diastasis reduction plays a more relevant role than fragment angulation in influencing union after odontoid screw placement for Anderson type II fractures. The fracture angulation and diastasis were measured on the pre- and postoperative CT scans. Patients were subcategorized into favorable and unfavorable for anterior odontoid screw placement, according to the fracture angulation $\left(>10^{\circ}\right.$ or $\left.<10^{\circ}\right)$. Although it has been reported that anterior screw placement should be avoided in patients with unfavorable fracture angulation, our daily experience seems to suggest that misalignment may represent a relative contraindication, strictly according to single-surgeon experience. An unfavorable angulation could result in a more challenging screw placement, which may be managed with careful preoperative surgical planning and proper patient positioning. According to our results, it appears that the fracture angulation does not influence the union rate. On the other hand, preoperative diastasis can be considered a radiological parameter when planning surgery, while the postoperative diastasis appears to influence the union rate.

Complications in our series amounted to $9.41 \%$, and 5 $(62.5 \%)$ of the 8 patients spontaneously recovered from transitory dysphagia, whereas the 3 patients reporting peri-implant radiolucency were followed up and did not report segmental instability; thus, no additional treatments were needed.

\section{Limitations}

Our investigation has some limitations we should disclose for a proper data interpretation. The relatively small sample of patients could have influenced subgroup analysis and the role of individual factors in influencing the union rate. There is no control group; thus, our data should be carefully evaluated, and comparative evaluations in future clinical prospective investigations are needed to confirm our results. Data on compliance with wearing the nonrigid cervical collar after surgery were not available, and this could have influenced the chances of bone union. Surgical procedures were conducted by different surgeons from the institutions involved in this study.

\section{Conclusions}

Our investigation suggests that duration from injury to surgery $>7$ days may be the most relevant factor influencing union rate after odontoid screw placement for Anderson type II fractures, followed by age $>70$ years, residual fragment diastasis $>1 \mathrm{~mm}$, osteoporosis, female gender, and diabetes. Timing of surgery could have been underestimated for odontoid fractures, although it has already been reported as relevant in other fractures. Accordingly, whenever surgery is selected as treatment, it should be performed within 1 week from injury. Furthermore, surgery after unsuccessful conservative management might not be as effective as an early procedure; thus, treatment selection should be fastidiously conducted. Prospective comparative studies are needed to confirm our results.

\section{References}

1. De Bonis P, Iaccarino C, Musio A, et al. Functional outcome of elderly patients treated for odontoid fracture: a multicenter study. Spine (Phila Pa 1976). 2019;44(13):951-958.

2. Gallie WE. Fractures and dislocations of the cervical spine. Am J Surg. 1939;46(3):495-499.

3. Joaquim AF, Patel AA. Surgical treatment of Type II odontoid fractures: anterior odontoid screw fixation or posterior cervical instrumented fusion? Neurosurg Focus. 2015;38(4): E11.

4. Anderson LD, D'Alonzo RT. Fractures of the odontoid process of the axis. J Bone Joint Surg Am. 1974;56(8):16631674.

5. Kepler CK, Vaccaro AR, Dibra F, et al. Neurologic injury because of trauma after type II odontoid nonunion. Spine $J$. 2014;14(6):903-908.

6. Fan L, Ou D, Huang X, et al. Surgery vs conservative treatment for type II and III odontoid fractures in a geriatric population: a meta-analysis. Medicine (Baltimore). 2019;98(44): e10281.

7. Nourbakhsh A, Shi R, Vannemreddy P, Nanda A. Operative versus nonoperative management of acute odontoid Type II fractures: a meta-analysis. J Neurosurg Spine. 2009;11(6): 651-658.

8. Yang Z, Yuan Z-Z, Ma J-X, Ma X-L. Conservative versus surgical treatment for type II odontoid fractures in the elderly: grading the evidence through a meta-analysis. Orthop Traumatol Surg Res. 2015;101(7):839-844.

9. Böhler J. Anterior stabilization for acute fractures and nonunions of the dens. J Bone Joint Surg Am . 1982;64(1):18-27.

10. Ricciardi L, Montano N, D'Onofrio GF, et al. X-ray exposure in odontoid screwing for Anderson type II fracture: comparison between $\mathrm{O}$-arm and $\mathrm{C}$-arm-assisted procedures. Acta Neurochir (Wien). 2020;162(3):713-718.

11. Shen Y, Miao J, Li C, et al. A meta-analysis of the fusion rate from surgical treatment for odontoid factures: anterior odontoid screw versus posterior C1-C2 arthrodesis. Eur Spine J. 2015;24(8):1649-1657.

12. Lofrese G, Musio A, De Iure F, et al. Type II odontoid fracture in elderly patients treated conservatively: is fracture healing the goal? Eur Spine J. 2019;28(5):1064-1071. 
13. Huybregts JGJ, Jacobs WCH, Vleggeert-Lankamp CLAM. The optimal treatment of type II and III odontoid fractures in the elderly: a systematic review. Eur Spine J. 2013;22(1):1-13.

14. Gembruch O, Lemonas E, Ahmadipour Y, et al. Treatment of odontoid type II fractures in octogenarians: balancing two different treatment strategies. Neurospine. 2019;16(2): 360-367.

15. Gembruch O, Lemonas E, Ahmadipour Y, et al. Nonoperative management of $\mathrm{C}-2$ dens fractures: single center experience and review of the literature. Clin Neurol Neurosurg. 2018;169:166-173.

16. Löhrer L, Raschke MJ, Thiesen D, et al. Current concepts in the treatment of Anderson Type II odontoid fractures in the elderly in Germany, Austria and Switzerland. Injury. 2012; 43(4):462-469.

17. Charles YP, Ntilikina Y, Blondel B, et al. Mortality, complication, and fusion rates of patients with odontoid fracture: the impact of age and comorbidities in 204 cases. Arch Orthop Trauma Surg. 2019;139(1):43-51.

18. Lofrese G, Cultrera F, Visani J, et al. Intraoperative Doppler ultrasound as a means of preventing vertebral artery injury during Goel and Harms C1-C2 posterior arthrodesis: technical note. J Neurosurg Spine. 2019;31(6):824-830.

19. Rao G, Apfelbaum RI. Odontoid screw fixation for fresh and remote fractures. Neurol India. 2005;53(4):416-423.

20. Lauretti WJ. The safety and effectiveness of common treatments for whiplash. In: Gatterman MI, ed. Whiplash. Mosby; 2012:169-178.

21. Ricciardi L, Scerrati A, Olivi A, et al. The role of cervical collar in functional restoration and fusion after anterior cervical discectomy and fusion without plating on single or double levels: a systematic review and meta-analysis. Eur Spine J. 2020;29(5):955-960.

22. Ricciardi L, Stifano V, D'Arrigo S, et al. The role of nonrigid cervical collar in pain relief and functional restoration after whiplash injury: a systematic review and a pooled analysis of randomized controlled trials. Eur Spine J. 2019; 28(8):1821-1828.

23. Polli FM, Miscusi M, Forcato S, Raco A. Atlantoaxial anterior transarticular screw fixation: a case series and reappraisal of the technique. Spine J. 2015;15(1):185-193.

24. Khattab MF, Nageeb Mahmoud A, Saeed Younis A, ElHawary Y. A simple technique for easier anterior odontoid screw fixation. Br J Neurosurg. 2019;33(2):135-139.

25. Hadley MN, Walters BC, Grabb PA, et al. Guidelines for the management of acute cervical spine and spinal cord injuries. Clin Neurosurg. 2002;49:407-498.
26. Artoni C, Pogliacomi F, Guardoli L, et al. Pelvic ring fractures: what about timing? Acta Biomed. 2019;90(12-S): $76-81$.

27. Jazayeri HE, Khavanin N, Yu JW, et al. Does early repair of orbital fractures result in superior patient outcomes? A systematic review and meta-analysis. J Oral Maxillofac Surg. 2020;78(4):568-577.

28. Lu S, Du T, Sun Z, et al. Timing of extremity fracture fixation in patients with traumatic brain injury: a meta-analysis of prognosis. World Neurosurg. 2020;133:227-236.

29. Papakostidis C, Panagiotopoulos A, Piccioli A, Giannoudis PV. Timing of internal fixation of femoral neck fractures. A systematic review and meta-analysis of the final outcome. Injury. 2015;46(3):459-466.

30. Apfelbaum RI, Lonser RR, Veres R, Casey A. Direct anterior screw fixation for recent and remote odontoid fractures. $J$ Neurosurg. 2000;93(2 Suppl):227-236.

\section{Disclosures}

Dr. Barrey reports being a consultant to Medicrea, Noraker, Global'S, and Implanet, and having a nonfinancial relationship to academic competition of the European Spine Journal Advisory Board.

\section{Author Contributions}

Conception and design: Ricciardi, Lofrese, Barrey, Piazza, Miscusi, Raco. Acquisition of data: Ricciardi, Trungu, Scerrati, Rustemi, Mazzetto, Lofrese, Cultrera, Di Bartolomeo, Piazza. Analysis and interpretation of data: Ricciardi, Scerrati, Miscusi. Drafting the article: Ricciardi, Trungu, Di Bartolomeo, Piazza. Critically revising the article: Scerrati, De Bonis, Lofrese, Barrey, Miscusi, Raco. Reviewed submitted version of manuscript: all authors. Approved the final version of the manuscript on behalf of all authors: Ricciardi. Statistical analysis: Ricciardi. Study supervision: De Bonis, Barrey, Miscusi, Raco.

\section{Correspondence}

Luca Ricciardi: UO di Neurochirurgia, Tricase, Lecce, Italy. ricciardi.lu@gmail.com. 